\title{
OPTOFLUIDICS
}

\section{Tunable droplet laser}

Appl. Phys. Lett. 107, 211105 (2015)

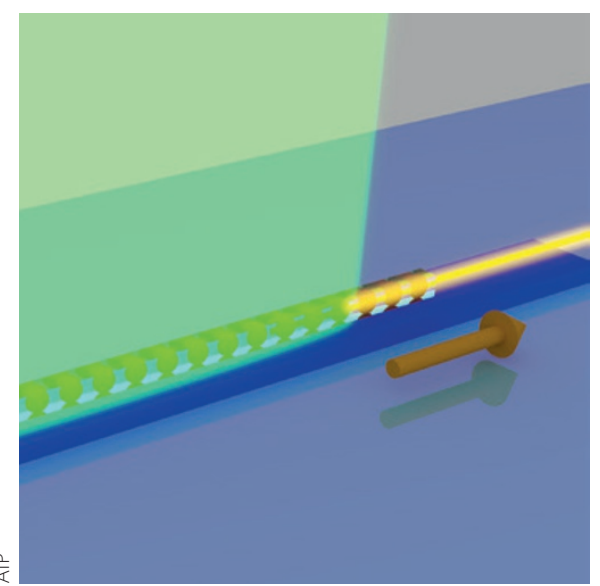

An on-chip optofluidic laser that is tunable across the visible wavelength band has been fabricated by scientists from Israel and Denmark. Avraham Bakal and co-workers from The Hebrew University of Jerusalem and the Technical University of Denmark made their laser from a periodic linear array of droplets of benzyl alcohol containing the dye R6G that are located inside a microfluidic channel filled with mineral oil. The droplets form due to the immiscibility of the alcohol and the oil. The R6G dye serves as an optical gain medium for the laser while refractive index corrugation associated with the droplet array acts like a distributed grating that provides optical feedback. When pumped with 532-nm nanosecond pulses from a frequency-doubled Nd:YAG laser, the device lases with the emission wavelength determined by the periodicity of the droplet array. The team demonstrated lasing that is tunable between about $570 \mathrm{~nm}$ and $640 \mathrm{~nm}$ by simply changing the pressure of the oil and alcohol reservoirs that feed the device. $O G$

\section{ENDOSCOPY}

\section{Two-photon imaging Opt. Express 23, 32158-32170 (2015)}

An endoscope typically uses multicore fibres (MCFs) to visualize the interior of the body. Unfortunately, MCFs deliver pixelated images due to the gap between the cores (3-5 $\mu \mathrm{m})$. Now, Edgar Morales-Delgado and colleagues from École Polytechnique Fédéral de Lausanne in Switzerland demonstrate high-resolution two-photon excitation imaging through a $20-\mathrm{cm}$-long graded-index multimode fibre (MMF) of $350 \mu \mathrm{m}$ diameter. In their approach, a focused $120 \mathrm{fs}$ pulse is delivered and scanned over the sample, and digital phase conjugation is performed using mode selection by time-gating with an ultrafast reference pulse. The excited two-photon emission is collected through the same fibre. Scanning the focused pulse in a $3 \mathrm{D}$ volume over a sample consisting of fluorescent beads suspended in a polymer achieves lateral and axial resolutions of $1 \mu \mathrm{m}$ and $15 \mu \mathrm{m}$, respectively. The team says that the high resolution, small probe diameter, large collection efficiency and sectioning capability opens the possibility for in vivo minimally invasive multiphoton endoscopy in the brain, the ear or the eye, for example. $R W$

\section{QUANTUM INFORMATION}

\section{Reconfigurable circuits \\ Light Sci. Appl. 4, e354 (2015)}

Fulvio Flamini and co-workers from Italy have now developed a reconfigurable integrated optical circuit by using an aluminoborosilicate glass substrate, and investigated the performance of the circuit in the quantum regime. The waveguide circuits for a tunable Mach-Zehnder interferometer were directly inscribed in the aluminoborosilicate glass by tightly
The difficulty in observing random, non-repetitive weak signals that are hidden in noise has caused detection challenges in astronomy, spectroscopy, biology and remote sensing. Now, a team from the University of California at San Diego, USA show that a fast, randomly occurring event can be successfully detected and extracted from a noisy background. Their approach uses instantaneous spectral cloning and a single-step, coherent field processor. More specifically, an 80-ps-long pulse was spectrally cloned and processed to increase the received signal-to-noise ratio by $14.1 \mathrm{~dB}$. To separate signal from noise, the number of spectral copies was varied from 6 to 36 , proving that background rejection can be progressively increased even when the random event is immersed in a high level of background noise. The team shows that an isolated 80 -ps pulse buried in noise could be detected with a confidence level exceeding $99 \%$. The team says that spectral cloning can be used for more complex analyses of random events, in both the microwave and optical domains. focused laser pulses at a wavelength of $1,030 \mathrm{~nm}$ and a duration of $300 \mathrm{fs}$. The fabricated waveguides yielded single-mode operation at $1,550 \mathrm{~nm}$ and propagation losses of $\sim 0.6 \mathrm{~dB} \mathrm{~cm}^{-1}$. The coupling losses to standard single-mode telecom fibres were $0.2 \mathrm{~dB}$ per facet. The phase in the interferometer was thermally controlled by tuning the voltage on a gold electrode on the glass substrate. Pairs of horizontally polarized identical photons were generated at a wavelength of $1,550 \mathrm{~nm}$ via spontaneous parametric down-conversion in a bismuth borate crystal and sent to the circuits. Tunable Hong-Ou-Mandel interference and superresolved fringes based on N00N states were successfully demonstrated with two-photon input states.

\section{LIGHT-MATTER INTERACTION}

\section{Nano-optical isolator}

Phys. Rev. X 5, 041036 (2015)

Optical isolators offering excellent performance are commercially available, but tend to be bulky devices on the scale of centimetres. Miniaturized isolators have been demonstrated but achieving high levels of isolation at nanoscale dimensions with very low light levels remains challenging. Now, Clément Sayrin and colleagues from the Vienna Center for Quantum Science and Technology in Austria have realized a nanoscale optical isolator using optical waveguides and cold atoms. The team used low-loss silica waveguides and demonstrated non-reciprocal transmission controlled via the internal state of spin-polarized atoms. And, importantly, their devices showed good performance in the single-photon regime. In the first part of the experimental work, cesium atoms were kept a distance of $230 \mathrm{~nm}$ from a nanofibre surface by an optical trapping scheme and 852-nm wavelength light was sent through the fibre to overlap with an atomic transition of the cesium atoms. Although interaction with a single atom is expected to result in only a weak, $\sim 0.3 \mathrm{~dB}$, value of isolation, the team showed that the use of a few tens of atoms enabled a much higher level of isolation of around $8 \mathrm{~dB}$. While increasing the number of atoms is one approach to increase isolation, improving the atom-light interaction strength is another. To do this the authors coupled a whispering-gallery-mode bottle microresonator to the fibre. This resulted in an experimentally demonstrated $13 \mathrm{~dB}$ of isolation using a single $\mathrm{Rb}$ atom coupled to the resonator mode.

Written by Oliver Graydon, Noriaki Horiuchi, David Pile and Rachel Won. 